\title{
O papel da equipe de enfermagem diante do contexto da COVID-19: revisão
}

\section{integrativa}

\author{
The role of the nursing team in the context of the COVID-19: integrative review \\ El papel del equipo de enfermería en el contexto del COVID-19: revisión integrativa
}

Recebido: 19/11/2021 | Revisado: 24/11/2021 | Aceito: 25/11/2021 | Publicado: 25/11/2021

Sueila Chaves dos Santos Fernandes ORCID: https://orcid.org/0000-0002-7223-8013 Faculdade Uniceuma, Brasil

E-mail: sueilachaves@live.com

Patrícia dos Santo Silva Queiroz

ORCID: https://orcid.org/0000-0002-9587-1786 Faculdade Unisulma, Brasil E-mail: patriciasqueiroz@gmail.com Aline de Sousa Rocha

ORCID: https://orcid.org/0000-0002-8181-0835 Faculdade de Imperatriz, Brasil E-mail: lillydarocha@hotmail.com

Benedita Maryjose Gleyk Gomes ORCID: https://orcid.org/0000-0002-5231-3936

Universidade Federal do Maranhão, Brasil E-mail: bene.belo@outlook.com

Cristina Limeira Leite

ORCID: https://orcid.org/0000-0002-7321-1496 Universidade do Estado do Rio de Janeiro, Brasil E-mail: crislimeira@gmail.com

Marluce Sampaio Nobre Barbosa ORCID: https://orcid.org/0000-0001-7276-6521 Universidade Federal do Pará, Brasil E-mail: marluce.nobre@ceuma.br

Haigle Reckziegel de Sousa

ORCID: https://orcid.org/0000-0002-5803-2289

Universidade de Santa Cruz do Sul, Brasil E-mail: haiglereck3@gmail.com

Gabriela Caroline Lima Júnior

ORCID: https://orcid.org/0000-0003-4115-9176 Faculdade Uniceuma, Brasil E-mail: gabqrzz@gmail.com Janayna Araújo Viana

ORCID: https://orcid.org/0000-0002-8855-5056 Faculdade do Bico do Papagaio, Brasil E-mail: janaynavi@hotmail.com

Francisco Alves Lima Júnior

ORCID: https://orcid.org/0000-0002-3117-4949

Universidade do Estado do Maranhão, Brasil

E-mail: francisco.enfdotrabalho@gmail.com

\section{Resumo}

O presente estudo tem a finalidade abordar qual o papel da equipe de enfermagem diante do contexto da COVID-19: e identificar de modo geral. Método: Trata-se de uma revisão de literatura por meio de investigação bibliográfica e para a construção deste estudo foi corporizada uma procura nas bases de dados da Biblioteca Virtual em Saúde,como: Literatura Latino-Americana e do Caribe em Ciências da Saúde (LILACS) eScientific Electronic Library Online (SciELO), empregando os descritores e suascombinações na língua portuguesa adequadas a base DECS (Descritores em Ciências da Saúde): COVID-19; Profissionais de Enfermagem; Pandemia. Resultados: os resultados evidenciam que o enfermeiro esta presente em toda a linha de cuidado ao paciente com COVID-19, no serviço de Urgência e Emergência estando responsável pela classificação e mensuração do risco, nas UTI's, cuidando de condições mais críticas do paciente, o que exige conhecimento e ciência das condutas e claro, no nível de atenção primária, onde por meio da consulta de enfermagem ele pode direcionar esse paciente a tratamentos específicos ou ainda acompanhar os casos diagnosticados como leves e simples da doença. Conclusão: ao final fica evidente a importância e relevância dos cuidados da equipe de enfermagem, por ser uma profissão multifacetada e por possuir sua atuação pautada na 
Sistematização da Assistência. O que norteia suas práticas e toda a tomada de decisão, o enfermeiro atua nos mais diferentes cenários e é inclusive pensado como ponto forte no combate às epidemias e pandemias, toda via, convive com um cenário que o faz enfrentar grandes dores, pelo isolamento social e familiar, bem como pelas condições inapropriadas de trabalho, para que este possa exercer o cuidado de forma digna e segura, uma vez que é indissociável da linha de frente contra a COVID-19.

Palavras-chave: COVID-19; Profissionais de enfermagem; Pandemia.

\begin{abstract}
The purpose of this study is to address the role of the nursing team in the context of the COVID-19: and to identify in general. Method: This is a literature review through bibliographical research and for the construction of this study a search has been embodied in the databases of the Virtual Library on Health, such as Latin American Literature and the Caribbean in Health Sciences (LILACS) and Scientific Electronic Library Online (SciELO),employing the descriptors and their combinations in the Portuguese language appropriate to the DECS base (Descriptors in Health Sciences): COVID-19;Nursing professionals; Pandemic. Results: results show that the nurse is present throughout the care line to the patient with COVID-19, in the emergency and emergency services being responsible for the classification and measurement of risk in the ICU, taking care of the most critical conditions of the patient, which requires knowledge and science of the conduits and of course, at the level of primary attention, where by means of nursing consultation he can direct this patient to specific treatments or even follow the cases diagnosed as mild and simple of the disease. Conclusion: In the end, it is evident the importance and relevance of nursing staff care, because it is a multifaceted profession and because it has its performance guided by the Sistematization of Assistance. What guides his practices and all decision-making, the nurse acts in the most different scenarios and is even thought of as a strong point in combating epidemics and pandemics, all the way through, coexists with a scenario that causes him to face great pain, social and family isolation, as well as inappropriate working conditions, to be able to exercise care in a dignified and safe way, since it is inseparable from the front line against COVID-19.
\end{abstract}

Keywords: COVID-19; Nursing professionals; Pandemic.

\title{
Resumen
}

El objetivo de este estudio fue explorar el papel del equipo de enfermería en la infección por COVID-19 y su identificación general. Métodos: Esta revisión bibliográfica se llevó a cabo a través de estudios bibliográficos, y para construir este estudio, se realizaron búsquedas en bases de datos de bibliotecas virtuales de salud, como la literatura latinoamericana y las ciencias de la salud del Caribe (lillacs) y la biblioteca electrónica de Ciencias en línea (scielo). Descriptor portugués y su combinación (descriptor de Ciencias de la salud) para la base de datos decs: nuevo COVID19; Profesionales de enfermería; Una pandemia. Resultados: los resultados mostraron que los enfermeros estaban presentes a lo largo de toda la línea de enfermería de los pacientes con COVID-19, responsables de la clasificación y medición de los riesgos en la UCI en los servicios de emergencia y emergencia, y de la atención a las situaciones más críticas de los pacientes, lo que requería conocimiento y ciencia del catéter, por supuesto. En el nivel de preocupación principal, a través de la consulta de enfermería, puede guiar al paciente en un tratamiento específico, e incluso rastrear los casos diagnosticados como enfermedades leves y simples. Conclusión: Por último, la importancia y pertinencia de la enfermería del personal de enfermería es obvia, ya que la enfermería del personal de enfermería es una profesión multifacética cuyo desempeño está guiado por la sistematización de la asistencia. Guiado por su práctica y por todas sus decisiones, el enfermero ha actuado en las situaciones más diversas, e incluso ha sido considerado un punto fuerte en la lucha contra las epidemias y epidemias, y ha coexistido durante mucho tiempo con las condiciones que lo han llevado a enfrentar enormes sufrimientos, aislamiento social y familiar y condiciones de trabajo inadecuadas, La capacidad de prestar atención de manera digna y segura es inseparable de la primera línea de la lucha contra el nuevo COVID-19.

Palabra clave COVID-19; Profesionales de enfermería; Pandemia.

\section{Introdução}

Em meados de dezembro de 2019, a doença intitulada COVID-19 (coronavírus) foi confirmada inicialmente na cidade de Wuhan, China. A partir de então, o número de casos em todo o mundo expandiu de forma alarmante. Por causa dessa lépida propalação, em que se propaga de pessoa para pessoa por meio de gotículas e aerossóis, tornou-se o que na epidemiologia é definido como pandemia. Destarte, por escassez de especialização em tratamento ou vacinas, a doença é abalizada como emergência de saúde pública e estada de alerta precoce (Taets et al., 2020). 
Esse novo vírus pertence à família do Coronaviridae pode infectar mamíferos e causar uma síndrome respiratória, podendo ser transmitida por pessoas infectadas em até sete dias logo após o início da sintomatologia ou quando a pessoa estiver assintomática (Thomas, 2020).

A revista Nature publicou um estudo onde relatou que a patologia da COVID - 19 ganhou tal proporção que perdeu a capacidade de ser controlada, desse modo, precisa-se de um feedback globalmente coordenada e que seja crucial organizar os sistemas de saúde para o enfrentamento da patologia do coronavírus (Callaway, 2020).

Durante o processo de combate da COVID-19 nas instituições de saúde, é fundamental uma grande equipe de profissionais da saúde. Dentre eles, os profissionais de enfermagem que somente no Brasil, existem cerca de 2,2 milhões, eles são considerados como protagonistas da linha de frente na assistência fornecida e necessitam não apenas de conhecimentos técnicos, mas também científicos. (Miranda et al., 2020).

$\mathrm{O}$ cuidado e assistência integral da equipe de enfermagem aos pacientes se encontram em todos os níveis da atenção hospitalar e são de extrema importância ao paciente acometido pelo vírus. Esses profissionais estão presentes desde a admissão do paciente até a alta hospitalar, além de obterem a característica de estarem presentes em todos os momentos e fases da vida do paciente. É relevante enfatizar o papel desses profissionais no cuidado, pois os mesmos cumprem o destaque no enfrentamento da COVID-19 (Queiroz et al., 2020).

Nesse sentido, o presente estudo tem a finalidade abordar qual o papel da equipe de enfermagem diante do contexto da covid-19: revisão integrativa e identificar de modo geral, quais as principais manifestações sofridas pelos pacientes com diagnostico da doença.

O artigo está disposto em sessões, que contemplam o referencial teórico onde é tratada a literatura de determinação da o papel da equipe de enfermagem diante do contexto da covid-19: revisão integrativa, em seu contexto total, as sessões seguintes tratarão a metodologia utilizada para a elaboração do artigo e os resultados e discussões como produto de todo o conteúdo escrito, por fim as considerações finais, encerrando a ideia central e sancionando os pontos de maior relevância encontrados ao longo desta produção.

\section{Referencial Teórico}

\subsection{Caracterização da COVID-19}

A sintomatologia da COVID-19 são extensos e múltiplos e seu surgimento pode variegar desde formas assintomáticas às pneumonias graves ou até mesmo levar ao óbito. No decorrer do estudo da WHO - CHINA que incluiu 55924 pacientes testificados positivamente para a doença, cerca de $80 \%$ deles apresentou a patologia de maneiera leve a moderada, $13,8 \%$ apresentaram de maneira severa que variou de: dispneia, frequência respiratória $\geq 30$ / minuto, saturação de oxigênio $\leq 93 \%$, $\mathrm{Pa} 02 / \mathrm{FiO} 2 \mathrm{FiO} 2$, ratio < 300 e/ou infiltrado pulmonar > 50\% do tecido pulmonar em 24/48 horas) e 6,1\% chegaram a estado críticos possuindo desde de insuficiência respiratória aguda à choque séptico e falência múltiplas de órgãos. (Aylward \& Liang, 2019).

A OMS - Organização Mundial da Saúde propalou a COVID-19 como uma pandemia e como finalização, qualquer sujeito com sinal de infecção respiratória deveria ser considerado potencialmente infectado pelo SARS-CoV-2. A pesquisa é baseada no teste RT-PCR de amostra de swab naso e orofaríngeo e de vias aéreas inferiores. Carecendo ser reforçado através de uma nova amostra do trato respiratório no momento que ocorrer dissensão com o quadro clínico avaliado. Caso o resultado for positivo de RT-PCR é fundamental que se comece o tratamento através do protocolo e precauções exibidas (Fernandes et al., 2019). 
Relacionado às circunstâncias da COVID-19, a complicação expande, uma vez que boas partes das variações não são tão conhecidas. Os sinais e sintomas tem obtido uma variedade diversificada das estirpes já identificadas do vírus, tornando-se cada vez mais frequente a necessidade de estudos e pesquisa no que tange os novos achados da doença (Taets et al, 2020).

Os sinais clínicos da COVID-19 vão desde condição de assintomáticos até a Síndrome do Desconforto Respiratório Agudo e alterações de vários órgãos. Dentre os sintomas mais comuns da doença podem ser citados: Fadiga, tosse seca, dispneia e fadiga. Não obstante, outros sintomas podem variar em menores periocidades, como mal-estar, náusea, diarreia, vômito conjuntivite, mialgia, dores de garganta, convulsão, dores no abdome e tórax, além de anosmia, ageusia e hiposmia. Vale evidenciar que os indivíduos em condições assintomáticas também representam uma grande quantidade dos infectados (Da Silva et al, 2020).

No que diz respeito a permanência dos sintomas, as informações colhidas e relatadas é que o surgimento desde os primeiros sintomas e hospitalização varia entre 2 a 8 dias, porém o tempo notado desde o princípio desses sintomas até a carência de ventilação mecânica invasiva (VMI) e até o óbito se deu em média de 11 e 23 dias (Leung et al, 2004).

Os sinais clínicos da COVID-19 vão desde condição de assintomáticos até a Síndrome do Desconforto Respiratório Agudo e alterações de vários órgãos. Dentre os sintomas mais comuns da doença podem ser citados: Fadiga, tosse seca, dispneia e fadiga. Não obstante, outros sintomas podem variar em menores periocidades, como mal-estar, náusea, diarreia, vomito conjuntivite, mialgia, dores de garganta, convulsão, dores no abdome e tórax, além de anosmia, ageusia e hiposmia. Vale evidenciar que os indivíduos em condições assintomáticas também representam uma grande quantidade dos infectados (da Silva et al., 2020).

No ângulo técnico, é preciso manifestar que a história do SARS-CoV-2, independente de curta é surpreendentemente uma verdadeira incógnita. Diversa analise de relatos têm sido propagadas, em vários lugares do mundo, mas ainda persistem infinitas incertezas. O que se sabe é que o maior número de letalidade e péssimo prognóstico da patologia são em pessoas idosas, porém aqueles maiores de 80 anos de idade. Tem-se retratado, semelhantemente, que sujeitos com comorbidades como cardiopatas, hipertensos, diabéticos, câncer e imunodeprimidos - possuem os prognósticos, com letalidades mais elevadas, como foi observado por Wu e Mcgoogan (2020), na China, Itália e também nos Estados Unidos (Siqueira-Batista et al, 2020).

Inúmeros tipos de Coronavírus tem a potencialidade de infectar seres humanos, provocando transformações respiratórias graves. O SARS - CoV-2 é o responsável da COVID - 19. Essa mesma patologia se espalhou por todo mundo e somente em 11 de março de 2020, a OMS - Organização Mundial de Saúde decretou que o então surto havia se tornado uma pandemia. Durante a escrita deste material (setembro, 2021), já havia cerca de mais de 8.085 .932 milhões de casos confirmados em 188 países e com cerca de mais de 348.399 de mortes confirmadas no mundo devido à COVID - 19 (Guimarães, 2020).

\subsection{Atuação da equipe de enfermagem diante da covid-19}

Hordineamente, por causa do momento pandêmico, está indubitável a importância do trabalho da enfermagem e, mais importante, a sociedade está cada vez mais reconhecendo e valorizando o trabalho da enfermagem. Pesquisas vêm sendo realizadas por enfermeiros, a atuação tem sido cada vez mais humanizada nos serviços, atendimentos de complexidade, os enfermeiros juntamente com a sua equipe vêm orientando a população através de medidas preventivas de saúde, por fim, percebe-se que o espaço ocupado pela equipe de enfermagem é imprescindível para enfrentamento da pandemia da COVID -19 (Soares, et al., 2020). 
O trabalho da equipe de enfermagem é abstruso, diverso e plurifacetado. Esses trabalhadores que integram essa categoria profissional adentram nos quatro principais setores do mercado de trabalho sendo eles: - (público, privado, filantrópico e educacional) e participam de diferentes níveis dos processos de atenção à saúde, que vai desde atenção básica a níveis de alta complexidade tecnológica. Durante a execução de suas atividades diárias, esses trabalhadores estão constantemente expostos a uma série de riscos ocupacionais: físicos, químicos, biológicos, mecânicos e de acidentes, ergonomia e psicossociais (Souza, et al., 2014).

Atuando na linha de frente da luta contra a COVID - 19, a equipe de enfermagem segue exercendo de acordo com diretrizes, princípios ontológicos e regulamentos. Fazendo o uso de sua autonomia com diligência e mensurando os contratempos que surgem no decorrer da jornada assistencial juntamente das instituições médicas de saúde e equipes profissionais na assistência que será prestada ao paciente (Brito Simonvil \& Giotto, 2020).

Compreende-se, portanto que a enfermagem atua diretamente desde promoção á saúde ate a linha de frente e nos diversos setores de atenção a saúde. No entanto, durante a vivência posta pela a pandemia da COVID - 19, o papel da enfermagem ficou em evidencia por todas as mídias existentes, exibindo assim todas as situações que vai desde adoecimento e riscos de vida devido às condições de trabalho (Oliveira et al, 2021).

O papel da equipe de enfermagem obtém ênfase por estarem diretamente relacionados com a assistência aos pacientes que se encontram infectados, em diversos contextos da saúde. Seja ela no pronto-socorro, em uma unidade de internação hospitalar ou ate mesmo em uma UTI, esses profissionais desempenham o seu papel juntamente com as demais equipes de saúde, e por diversas vezes sem os artifícios que precisam para exercer o cuidado prestado com excelência (de Souza, Faustino $\&$ da Cruz, 2020).

\section{Metodologia}

Trata-se de uma revisão de literatura por meio de levantamento bibliográfico, considerada um método que tem como finalidade recopilar resultados obtidos em pesquisas sobre uma temática ou questionamento, de forma ordenada, sistemática e holística (Galvão \& Pereira, 2014).

Este gênero de revisão aplica também para seu levantamento "aspectos conceituais e metodológicos de fontes essenciais e outras do gênero manuais, vídeos, livros, sites e anais de eventos", beneficiando obter e inovar o conhecimento sobre o estado da arte de uma temática particular. (Medeiros \& Teixeira, 2016).

A pesquisa foi efetuada em bibliotecas, bases de dados e portais no decorrer dos meses agosto á novembro de 2021. Para conceito dos estudos foram utilizados os descritores deliberados de acordo com a Biblioteca Virtual da Saúde (BVS): Profissionais de Enfermagem, Coronavírus, pacientes, COVID-19 e Pandemia. Os sites e base de dados utilizados foram: Biblioteca Virtual em Saúde (BVS), SCIELO, LILACS, BDENF, World Health Organization, Medline. Nos critérios de inclusão consideraram-se: publicações em base de dados científicos disponíveis em português, inglês e espanhol, fluxogramas, checklist, guias, protocolos e recomendações publicados em sites governamentais. Já nos critérios de exclusão foram eliminados artigos e documentos que não correlacionavam com a temática a ser tratada nesta revisão. Nenhum recorte temporal foi estabelecido de forma a não delimitar os resultados a um determinado tempo.

Ao realizar a busca foram encontradas 35 publicações, das quais 16 contemplaram os critérios de inclusão. Posteriormente a leitura dos títulos e resumos, com intuito de elaborar o delineamento temático relacionado Atuação da equipe de enfermagem voltada a pacientes em tratamento da COVID - 19 sobraram 13 trabalhos científicos, os quais a base analítica do estudo. O desenvolvimento da analise aconteceu por meio da analise de conteúdo, sendo constituídas por três etapas: pré analise exploração do estudo e desenvolvimento e a critica dos resultados alcançado. 
A busca inicial resultou em 45 publicações, destas, 16 atenderam aos critérios de inclusão estabelecidos. Após a leitura dos títulos e resumos, a fim de realizar o recorte temático relacionado à assistência de enfermagem nos serviços de emergência hospitalar frente à COVID-19, restaram 12 documentos científicos, os quais constituem o corpus da análise do estudo. O processo de análise foi desenvolvido por meio da análise de conteúdo, em três etapas: a pré-análise, a exploração do material e o desenvolvimento e interpretação dos resultados obtidos.

A extração de dados é realizada por meio de estudos bibliográficos de vários trabalhos que enfatizam os objetos de pesquisa. Em suma, após a coleta dos dados, a análise começa sob a pesquisa narrativa, pois proporciona aos investigadores um contato ativo e direto com a base.

Figura 1. Fluxograma de recuperação dos artigos.

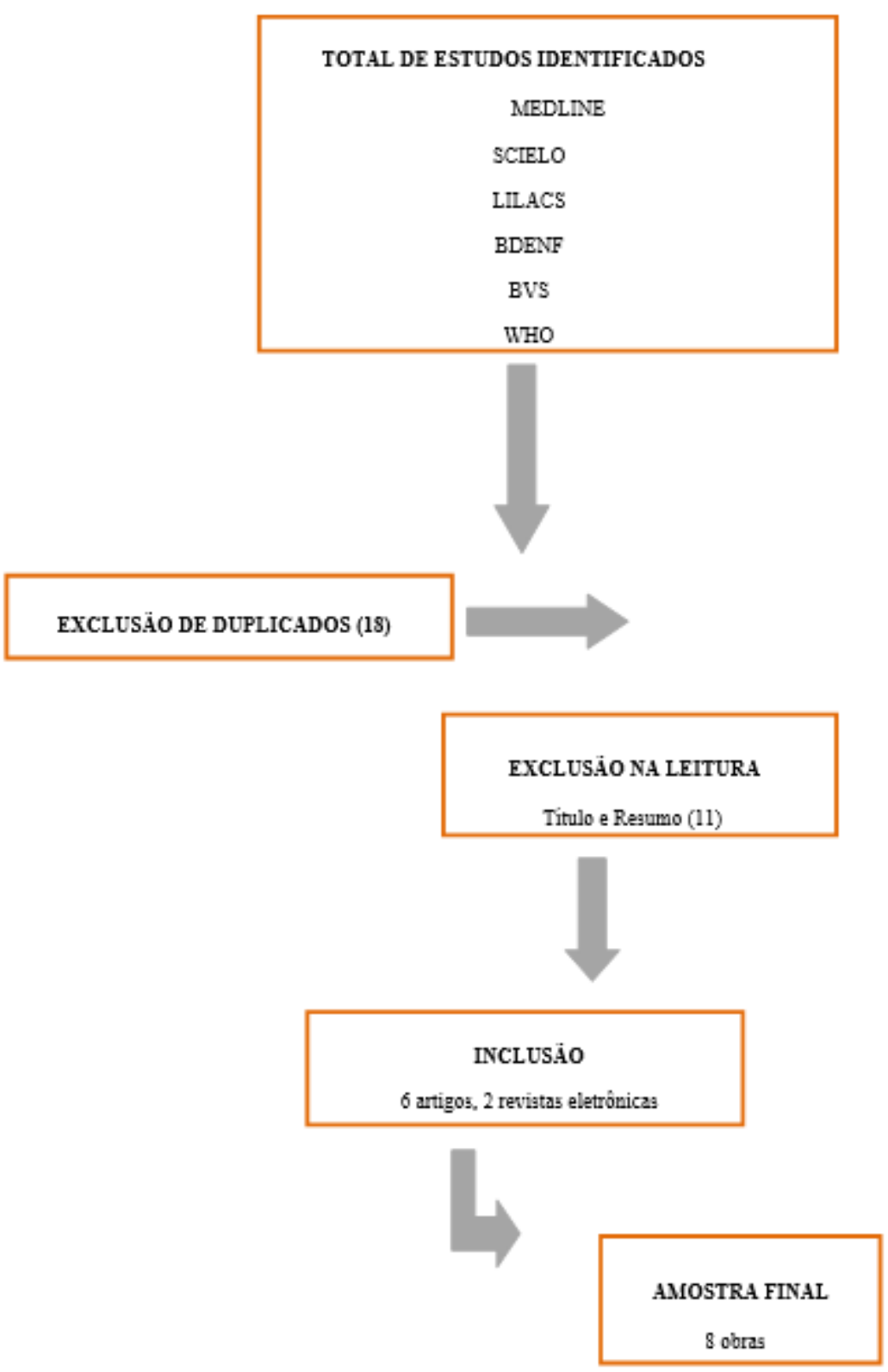

Fonte: Autores. 
A extração de dados é realizada por meio de estudos bibliográficos de vários trabalhos que enfatizam os objetos de pesquisa. Em suma, após a coleta dos dados, a análise começa sob a pesquisa narrativa, pois proporciona aos investigadores um contato ativo e direto com a base.

\section{Resultados e Discussão}

A amostra final desta revisão foi constituída por 17 (dezessete) trabalhos, selecionados pelos critérios de inclusão previamente estabelecidos. O Quadro 1 representa as especificações de cada um dos artigos, distribuídos, segundo: ano; periódico; nome dos autores e título e tipo de pesquisa.

Quadro 1. Artigos levantados para revisão de literatura.

\begin{tabular}{|c|c|c|c|}
\hline ANO & PERIÓDICO & AUTORES & TÍTULO \\
\hline 2020 & Health Sciences & $\begin{array}{l}\text { Taets, G., Barbosa, J., Bitencourt, G., \& } \\
\text { Taets, C. C }\end{array}$ & $\begin{array}{l}\text { Padrões funcionais de saúde em adultos com COVID-19 na } \\
\text { terapia intensiva: fundamentação aos diagnósticos de } \\
\text { enfermagem. }\end{array}$ \\
\hline 2020 & $\begin{array}{l}\text { Brazilian Journal of } \\
\text { Health Review }\end{array}$ & $\begin{array}{l}\text { Thomas, L. S., Pietrowski, K., da Silva } \\
\text { Kinalski, S., Bittencourt, V. L. L., \& } \\
\text { Sangoi, K. C. M. }\end{array}$ & $\begin{array}{l}\text { Atuação do enfermeiro emergencista na pandemia de } \\
\text { covid-19: Revisão narrativa da literatura. }\end{array}$ \\
\hline 2020 & Nature & CALLAWAY, E. & $\begin{array}{l}\text { Time to use the } \mathrm{p}-\text { Word? Coranavírus enters dangerous } \\
\text { new phase }\end{array}$ \\
\hline 2020 & Cogitare Enfermagem & $\begin{array}{l}\text { MIRANDA, Fernanda Moura D.'Almeida } \\
\text { et al. }\end{array}$ & $\begin{array}{l}\text { Condições de trabalho e o impacto na saúde dos } \\
\text { profissionais de enfermagem frente a Covid- } 19 .\end{array}$ \\
\hline 2020 & $\begin{array}{l}\text { Journal of Health \& } \\
\text { Biological Sciences }\end{array}$ & QUEIROZ, Amanda Gabrielle Silva et al. & $\begin{array}{l}\text { Diagnósticos de enfermagem segundo a taxonomia da } \\
\text { NANDA internacional para sistematização da assistência } \\
\text { de enfermagem a COVID- } 19 \text {. }\end{array}$ \\
\hline 2019 & $\begin{array}{l}\text { China Joint Mission on } \\
\text { Coronavirus Disease }\end{array}$ & AYLWARD, B; LIANG W. & $\begin{array}{l}\text { Reporto of the WHO - China Joint. Mission on } \\
\text { Coronavirus disease } 2019 .\end{array}$ \\
\hline 2020 & $\begin{array}{l}\text { Health Residencies } \\
\text { Journal-HRJ }\end{array}$ & FERNANDES, C.A. et al. & $\begin{array}{l}\text { Desafios e recomendações para o cuidado intensivo de } \\
\text { adultos críticos com doença de coronavírus } 2019 \text { (COVID- } \\
\text { 19). }\end{array}$ \\
\hline 2020 & $\begin{array}{l}\text { Brazilian Journal of } \\
\text { Health Review }\end{array}$ & DA SILVA, D. P. et al. & $\begin{array}{l}\text { Aspectos da infecção ocasionada pelo Coronavírus da } \\
\text { Síndrome Respiratória Aguda Grave } 2 .\end{array}$ \\
\hline 2020 & $\begin{array}{l}\text { WHO. World Health } \\
\text { Organization. }\end{array}$ & SIQUEIRA-BATISTA, R. et al. & $\begin{array}{c}\text { COVID-19 e o fim da vida: quem será admitido na } \\
\text { Unidade de Terapia Intensiva }\end{array}$ \\
\hline 2020 & $\begin{array}{c}\text { Fisioterapia em } \\
\text { Movimento. Epub. }\end{array}$ & GUIMARÃES, F. & $\begin{array}{l}\text { Atuação do fisioterapeuta em unidades de terapia intensiva } \\
\text { no contexto da pandemia de COVID- } 19 .\end{array}$ \\
\hline 2020 & Escola Anna Nery & SOARES, S.S. S. et al. & $\begin{array}{l}\text { De cuidador a paciente: na pandemia da Covid-19, quem } \\
\text { defende e cuida da enfermagem brasileira?. }\end{array}$ \\
\hline 2014 & $\begin{array}{l}\text { Revista Mineira de } \\
\text { Enfermagem, }\end{array}$ & SOUZA, N.V. D. de O. et al & $\begin{array}{c}\text { Riscos ocupacionais e agravos à saúde dos trabalhadores } \\
\text { em uma unidade ambulatorial especializada. }\end{array}$ \\
\hline 2020 & $\begin{array}{l}\text { Revista De Iniciação } \\
\text { Científica E Extensão }\end{array}$ & $\begin{array}{l}\text { BRITO, L. L.; SIMONVIL, S.; GIOTTO, } \\
\text { A. C. }\end{array}$ & $\begin{array}{l}\text { Autonomia do profissional de enfermagem diante da covid- } \\
\text { 19: revisão integrativa. }\end{array}$ \\
\hline 2021 & Rev. Gaúcha Enferm. & OLIVEIRA, K. K. D. et al. & $\begin{array}{l}\text { Nursing Now e o papel da enfermagem no contexto da } \\
\text { pandemia e do trabalho atual. }\end{array}$ \\
\hline 2020 & Enfermagem em Foco & $\begin{array}{l}\text { DE SOUZA, D. P. H. FAUSTINO, A. M.; } \\
\text { DA CRUZ, K. C. T. }\end{array}$ & $\begin{array}{l}\text { A enfermagem em destaque na pandemia da Covid-19: } \\
\text { uma análise em mídias sociais. }\end{array}$ \\
\hline 2014 & Epidemiol. Serv. Saúde & GALVÃO, T.F., PEREIRA, M.G. & $\begin{array}{l}\text { Revisões sistemáticas da literatura: passos para sua } \\
\text { elaboração. }\end{array}$ \\
\hline 2016 & Resenha de livro & MEDEIROS, H. P.; TEIXEIRA, E. & Metodologia da pesquisa para a enfermagem e saúde \\
\hline
\end{tabular}

Fonte: Autores.

Por se tratar de um estudo multifacetado, foram selecionados e trabalhados 17 artigos (destacados na tabela acima), desses 17 pelo menos 10 abordam especificamente a atuação da enfermagem no contexto pandêmico da COVID- 19, os textos variam em conteúdo desde a atuação e papel pontual do enfermeiro, ate as condições de trabalho e problemáticas vivenciadas 
por esses profissionais de extrema importância no combate ao vírus e que dada a circunstância de trabalho pode sofrer comprometimento nas suas atribuições.

Thomas et al., (2020), destacam em seu estudo a importância de serviços "porta de entradas" dos pacientes, como um local que necessita de organização e estratégia para o acolhimento de toda a demanda, de modo que o profissional enfermeiro faça das suas atribuições pontos de segurança, para um atendimento seguro desde a classificação de risco até o momento de uma possível internação e a continuidade do atendimento até a alta.

Para tanto, ainda seguindo as linhas de pensamento elaboradas por Thomas et al., (2020), fica evidente a necessidade do preparo do profissional enfermeiro, tanto na condição de gestor como de assistencialista, para que a prestação do cuidado ocorra com qualidade e resolutividade, por meio da escuta qualificada, raciocínio clinico e crítico, desenvolvimento técnico cientifico, dentre outras indispensáveis atribuições.

Queiroz et al., (2020), traz em seu estudo uma concretização do que é destacado pelos autores acima, toda via, ressalta que não somente no cenário da doença, mas que de modo global o enfermeiro pode ser compreendido como figura ativa e fundamental ao contexto do controle a epidemias e pandemias, destacando ainda que esse profissional também deve ser responsável pela integridade e segurança de sua equipe. A autora ainda coloca em evidencia a grande valia que existe na tomada de decisões do enfermeiro, com base solida na elaboração do Processo de enfermagem (PE), uma vez que o PE, fornece a enfermagem, assistência segura, base técnica, cientifica e assistência de qualidade, para a implementação das suas ações.

No estudo de Brito et. al., (2020), os autores salientam pontos importantes para debate e analise que consiste no cenário percebido e vivido pelo enfermeiro, destacando a sua exposição prolongada ao vírus, culminando, por conseguinte na exposição refletida nos seus familiares. Porem, semelhantemente a Queiroz et al., (2020), Brito et al., (2020) destaca a importância da prestação do cuidado da enfermagem, estruturada na Sistematização da Assistência de Enfermagem (SAE) e no Processo de enfermagem.

Para Taests (2020) as principais ações da enfermagem estão alocadas nos aspectos clínicos relacionados ao paciente, o autor relata a complexidade existente para a prestação do cuidado, dada a necessidade do paciente e pro vezes o manuseio de aparelhos e tecnologias diversas, objetivando maximizar a qualidade do cuidado. $\mathrm{O}$ autor pontua cuidados específicos que estão inerentes a atuação do enfermeiro, como por exemplo: passagem e manutenção de sondagem enteral; mensuração da infusão e eliminação de líquidos; observação e controle dos riscos de infecção; cuidados com a mobilidade do paciente no leito; cuidado com a ventilação do paciente, tendo como base a troca gasosa prejudicada, além da avaliação do padrão cognitivo dentre outros fatores.

Para Oliveira et. al., (2021) é importante mencionar que as instituições de saúde de todo o mundo sabem e devem reconhecer a importância de tal profissional, nessa guerra contra a COVID-19, profissionais que se fazem presente desde o diagnostico da doença até o cuidado hospitalar, como já mencionado acima por outros autores. Elencando ainda as mais variadas fragilidades as quais o enfermeiro está exposto, insumos e equipamentos insuficiente, colocando-os em constantes desafios de ordem profissional e pessoal, e mesmo diante do exposto, os profissionais seguiram de forma comprometida e ética na prestação do cuidado.

Mesmo a literatura destacando a importância do profissional enfermeiro no serviço hospitalar, quando na oferta do cuidado aos pacientes acometidos pela COVID- 19, mais uma vez se faz necessária a abordagem realizada por Brito et. al., (2020), lembrando-se da Atenção Primária a Saúde, local onde o enfermeiro atua na realização de consultas de enfermagem, solicitando exames complementares, além do encaminhamento a outros serviços caso seja necessário, além é claro do monitoramento dos casos leves diagnosticados no território. 


\section{Considerações Finais}

O estudo apresentado, que vislumbrava tratar sobre o papel da enfermagem contra a COVID- 19 apresenta êxito quando traz em seus resultados exatamente a abordagem relatada. É evidenciado nas falas dos mais variados autores, a problemática que permeia na atuação do enfermeiro frente a COVID- 19, evidenciadas pelo distanciamento familiar, serviços precários, ausência de materiais básicos para a sua proteção, porem mesmo diante de tais condições o enfermeiro seguiu os padrões éticos e humanísticos, ofertando cuidado integral e complexo ao individuo adoecido.

As atividades desempenhadas pelo enfermeiro abrangem as mais variadas funções, desde aquelas mais complexas relacionadas às unidades de Urgência e Emergência até aquelas relacionadas a Unidade de Terapia Intensiva (UTI), quando o enfermeiro precisa dispor de conhecimento técnico cientifico e habilidade para lidar com aparelhagem complexas, dele dependendo toda a linha de cuidado que envolve o paciente, como o manuseio no leito, sondagem para a nutrição, administração de medicaçães complexas e avaliação diária constante por meio da aplicação da sistematização da assistência de enfermagem, bem como pelo Processo de Enfermagem.

É importante destacar que o enfermeiro não possui atuação apenas nos serviços hospitalares, isso se faz evidente por autores que destacam a importância do enfermeiro na atenção primária a saúde, com o desenvolvimento da consulta de enfermagem e monitoramento residencial dos casos da doença que cursam com sintomas leves.

Para tanto, todos os materiais trabalhados se preocupam em abordar a magnitude que é a profissão de enfermagem, sendo a maior classe profissional na linha de frente contra a COVID-19 e por tanto, apresentando importância primordial no cuidado ao paciente, desde os casos simples aos complexos, principalmente quando o enfermeiro oferta a assistência de modo sistematizada e guiada, sabendo exatamente qual passo vem após o outro.

Em suma, diante das pesquisas envolvidas, é necessário encontrar e estabelecer novas pesquisas, principalmente para abordar O papel da equipe de enfermagem diante do contexto da covid-19: revisão integrativa. Portanto, pesquisas futuras podem trazer contribuições úteis para as evidências científicas, revelar inovações e cuidados atuais e ter como objetivo contribuir com a literatura científica e expandir novos conhecimentos.

\section{Referências}

Aylward, B. \& Liang. (2019). WHO China Joint Mission. Reporto of the WHO - China Joint. Mission on https://www.who.int/docs/defaultsource/coronaviruse/who-china-joint-mission-on-covid-19-final-report.pdf.

Brito, L. L., Simonvil, S., \& Giotto, A. C. (2020). Autonomia do profissional de enfermagem diante da covid-19: revisão integrativa. Revista De Iniciação Científica Extensão, 3(2), 420-37.

Callaway, E. (2020). Time to use the p-word? Coronavirus enters dangerous new phase. Nature (Lond.).

da Silva, D. P., dos Santos, I. M. R., \& dos Santos Melo, V. (2020). Aspectos da infecção ocasionada pelo Coronavírus da Síndrome Respiratória Aguda Grave 2 (SARS-CoV-2). Brazilian Journal of Health Review, 3(2), 3763-3779.

de Souza Domingues, P. H., Faustino, A. M., \& da Cruz, K. C. T. (2020). A enfermagem em destaque na pandemia da Covid-19: uma análise em mídias sociais. Enfermagem em Foco, 11(6).

Fernandes, C. A., da Cunha, A. M., Hemkemaier, E. C. R. C., do Carmo, K. D. S., Moraes, T. V. P., dos Santos, T. P., \& Mateus, N. C. (2020). Desafios e recomendações para o cuidado intensivo de adultos críticos com doença de coronavírus 2019 (COVID-19). Health Residencies Journal-HRJ, 1(1), 21-47.

Ferreira, A. M., Rocha, E. D. N. D., Lopes, C. T., Bachion, M. M., Lopes, J. D. L., \& Barros, A. L. B. L. D. (2016). Nursing diagnoses in intensive care: crossmapping and NANDA-I taxonomy. Revista brasileira de enfermagem, 69, 307-315.

Galvão, T. F., \& Pereira, M. G. (2014). Revisões sistemáticas da literatura: passos para sua elaboração. Epidemiologia e Serviços de Saúde, $23,183-184$.

Guimarães, F. (2020). Atuação do fisioterapeuta em unidades de terapia intensiva no contexto da pandemia de COVID-19.

Leung, G. M., Hedley, A. J., Ho, L. M., Chau, P., Wong, I. O., Thach, T. Q., \& Lam, T. H. (2004). The epidemiology of severe acute respiratory syndrome in the 2003 Hong Kong epidemic: an analysis of all 1755 patients. Annals of internal medicine, 141(9), 662-673.

Medeiros, H. P., \& Teixeira, E. (2016). Metodologia da pesquisa para a enfermagem e saúde: Resenha de livro. 
Research, Society and Development, v. 10, n. 15, e547101523587, 2021 (CC BY 4.0) | ISSN 2525-3409 | DOI: http://dx.doi.org/10.33448/rsd-v10i15.23587

Miranda, F. M. D. A., de Lima Santana, L., Pizzolato, A. C., \& Sarquis, L. M. M. (2020). Condições de trabalho e o impacto na saúde dos profissionais de enfermagem frente a Covid-19. Cogitare enfermagem, 25.

Oliveira, K. K. D. D., Freitas, R. J. M. D., Araújo, J. L. D., \& Gomes, J. G. N. (2020). Nursing Now e o papel da enfermagem no contexto da pandemia e do trabalho atual. Revista Gaúcha de Enfermagem, 42.

Queiroz, A. G. S., de Souza, R. Z., Sottocornola, S. F., Barbosa, S. J., Pinheiro, F. A., \& de Souza, L. P. (2020). Diagnósticos de enfermagem segundo a taxonomia da NANDA internacional para sistematização da assistência de enfermagem a COVID-19. Journal of Health \& Biological Sciences, 8(1), 1-6.

Siqueira-Batista, R., Gomes, A. P., Braga, L. M., Costa, A. D. S., Thomé, B., Schramm, F. R., Marinho, S. (2020). COVID-19 e o fim da vida: quem será admitido na Unidade de Terapia Intensiva?

Soares, S. S. S., Souza, N. V. D. D. O., Carvalho, E. C., Andrade, K. B. S. D., Pereira, S. R. M., \& Costa, C. C. P. D. (2020). De cuidador a paciente: na pandemia da Covid-19, quem defende e cuida da enfermagem brasileira? Escola Anna Nery, 24.

Souza, N. V. D. D. O., Pires, A. D. S., Gonçalves, F. G. D. A., Cunha, L. D. S., Ribeiro, L. V., \& Vieira, R. D. S. (2014). Riscos ocupacionais e agravos à saúde dos trabalhadores em uma unidade ambulatorial especializada. Revista Mineira de Enfermagem, 18(4), 923-938.

Taets, G., Barbosa, J., Bitencourt, G., \& Taets, C. C. Padrões funcionais de saúde em adultos com COVID-19 na terapia intensiva: fundamentação aos diagnósticos de enfermagem.

Thomas, L. S., Pietrowski, K., da Silva Kinalski, S., Bittencourt, V. L. L., \& Sangoi, K. C. M. (2020). Atuação do enfermeiro emergencista na pandemia de covid-19: Revisão narrativa da literatura. Brazilian Journal of Health Review, 3(6), 15959-15977. 